Shifted balance of dorsal versus ventral striatal communication with frontal reward and regulatory regions in cannabis dependence

Zhou Feng ${ }^{a *}$, M.S. Zimmermann Kaelib*, M.S., Xin Fei ${ }^{a}$, M.S., Dirk Scheele ${ }^{b}$, Ph.D., Wolfgang Dauc $^{c}$, M.S., Markus Banger ${ }^{c}$, M.D., Bernd Weber, , M.D., René Hurlemann' ${ }^{\text {b }}$ M.D., Ph.D., Keith M Kendrick ${ }^{a}$, Ph.D. Benjamin Becker ${ }^{a}$, Ph.D.

\title{
Affiliations
}

${ }^{a}$ The Clinical Hospital of Chengdu Brain Science Institute, MOE Key Laboratory for Neuroinformation, University of Electronic Science and Technology of China, 611731, Chengdu, China

a Department of Psychiatry and Division of Medical Psychology, University of Bonn, 53105 Bonn, Germany

'Department of Addiction and Psychotherapy, LVR-Clinic Bonn, 53111 Bonn, Germany ${ }^{\mathrm{d}}$ Center for Economics and Neuroscience, Department of Epileptology, University of Bonn ${ }^{e}$ Department of NeuroCognition, Life \& Brain Center, 53105 Bonn, Germany

\section{*equal contributions}

\section{Correspondence and reprint requests:}

Benjamin Becker

Key Laboratory for Neurolnformation

Center for Information in Medicine

University of Electronic Science and Technology

Chengdu 611731, China.

Tel: 86-28-61830811

mail: ben becker@gmx.de 


\section{ABSTRACT}

The transition from voluntary to addictive behavior is characterized by a loss of regulatory control in favor of reward driven behavior. Animal models indicate that this process is neurally underpinned by a shift in ventral to dorsal striatal control of behavior, however this shift has not been directly examined in humans. Against this background the present resting state fMRI study employed a two-step approach to (1) precisely map striatal alterations using a novel, data-driven network classification strategy combining Intrinsic Connectivity Contrast (ICC) with Multivoxel Pattern Analysis (MVPA) and, (2) to determine whether a ventral to dorsal striatal shift in connectivity with reward and and regulatory control regions can be observed in abstinent ( 28 days) male cannabis-dependent individuals $(n=24)$ relative to matched controls $(n=28)$. Network classification revealed that the groups can be reliably discriminated by global connectivity profiles of two striatal regions that mapped onto the ventral (nucleus accumbens) and dorsal striatum (caudate). Subsequent functional connectivity analysis demonstrated a relative shift between ventral and dorsal striatal communication with fronto-limbic regions that have been consistently involved in reward processing (rostral ACC) and executive / regulatory functions (dorsomedial PFC). Specifically, in the cannabis dependent subjects connectivity between the ventral striatum with the rostral ACC increased, whereas both striatal regions were uncoupled from the regulatory dorsomedial PFC. Together these findings suggest a shift in the balance between dorsal and ventral striatal control in cannabis dependence. Similar changes have been observed in animal models and may promote the loss of control central to addictive behavior. 


\section{INTRODUCTION}

Converging evidence from animal and human research indicates that maladaptations in fronto-limbic-striatal circuitries drive the loss of control that characterizes addiction (Fineberg et al., 2010; Morein-Zamir and Robbins, 2015). The striatum lies at the core of this circuitry and critically contributes to both, acute drug reinforcement and the transition from voluntary to addictive use that is accompanied by a loss of control (Brand et al., 2016; Everitt and Robbins, 2016; Fineberg et al., 2010).

The striatum contributes to several domains that undergo critical adaptations during the transition to addiction, including associative learning, behavioral control, incentive salience and reward (Haber, 2016; Robbins et al., 2012). The functional heterogeneity of the striatum and its multifaceted contributions to addiction are mirrored in its complex organization into distinct subregions that communicate with the entire cortex via subregion-specific fronto-striatal loops (Haber, 2016). Based on their specific functions and projections, the striatum is divided into the ventral striatum (VS), which includes reward processing hubs such as the nucleus accumbens, and the dorsal striatum (DS) that exhibits strong connections with dorsolateral and dorsomedial prefrontal regions engaged in regulatory control (Di Martino et al., 2008; Haber, 2016; Postuma and Dagher, 2006). This seggregation has important implications for the transition to addiction, with influential conceptualizations proposing that the loss of control is accompanied by a shift between ventral and dorsal striatal control of behavior (Everitt and Robbins, 2016; Jentsch and Taylor, 1999).

Partly driven by the ongoing discussion about cannabis legalization and concomitantly increasing demand for treatment of cannabis-dependence (EMCDDA, 2008; Volkow et al., 2016) a number of studies have examined effects of cannabis use on the brain (overview see e.g. Lorenzetti et al., 2016; Weinstein et al., 2016). In line with the functions of the striato-frontal circuitry and its proposed contribution to addiction task-based neuroimaging studies demonstrated exaggerated striatal reactivity to cannabis cues (Filbey et al., 2016), reward anticipation (Jager et al., 2013) and decreased frontal activity during executive control (Weinstein et al., 2016; Wrege et al., 2014; Yanes et al., 2018) in chronic users. This 
pattern mirrors mal-adaptations that have been observed across addictive disorders (Brand et al., 2016; Goldstein and Volkow, 2011).

Despite the important contributions of these studies to characterize mechanisms of cannabis dependence, the task-based approach does not allow direct evaluation of the ventral to dorsal striatal shift due to (1) limitation of task-based fMRI to regions engaged by the experimental paradigm (Weinstein et al., 2016), and (2) stimulus- and context-dependence of striatal alterations in cannabis dependence (Gilman, 2017; Zimmermann et al., 2017b). Resting state fMRI functional connectivity (rsfMRI-FC) approaches allow a more holistic assessment of functional alterations in the absence of task or contextual modulation. Moreover, these approaches have a high sensitivity for transient and lasting striatal alterations on the subregion-specific network level (Di Martino et al., 2011; Di Martino et al., 2008). Two recent studies applied rsfMRI-FC to determine lasting distruption in the fronto-striatal functional circuitries in cannabis dependence (Blanco-Hinojo et al., 2017; Zimmermann et al., 2017b). However, findings were limited by the confirmatory nature of the rsfMRI-FC analysis (Zimmermann et al., 2017b) or the use of literature-based striatal seed regions (Blanco-Hinojo et al., 2017) that have a high sensitivity to capture sub-region specific functional networks in healthy subjects (Di Martino et al., 2008) but might not be sensitive to the specific alterations related to cannabis dependence. Moreover, the ventral to dorsal shift has not been explicitly examined in these studies.

The present study therefore employed a two step approach to (1) precisely map striatal alterations in cannabis-dependent individuals using network classification and, (2) to determine whether a ventral to dorsal striatal shift in connectivity with reward and and regulatory control regions can be observed. In an initial step intrinsic striatal alterations were mapped using a novel fully data-driven network classification approach that operates independently of a priori assumptions about striatal architecture at the voxel level and thus enables higher sensitivity to detect striatal functional changes (Martuzzi et al., 2011; Rubinov and Sporns, 2010). Next, altered functional communication of the determined striatal subregions was examined on the whole-brain network level (for a similar approach see Walpola et al., 2017). To this rsfMRI data was acquired in $n=28$ participants that fulfilled the DSM-IV criteria for cannabis dependence and $n=28$ matched healthy controls. To control 
for subacute effects of cannabinoid metabolites (Vandevenne et al., 2000), craving

(Copersino et al., 2006) and rapid neural recovery of homeostatic receptor adaptations after cessation of chronic cannabis use (Hirvonen et al., 2012) participants underwent 28-days of cannabis abstinence before acquisition of the MRI data. Comparable abstinence periods have been employed in previous studies to differentiate enduring (long-term) effects of cannabis use from transient (sub-acute) alterations and rapid neural recovery (overview in Crean et al., 2011; Ganzer et al., 2016; Schreiner and Dunn, 2012).

Based on current neurobiological frameworks suggesting that the transition to addiction is accompanied by regional-specific neuroplastic changes in the ventral and dorsal striatum and accumulating evidence from task-based neuroimaging studies suggesting that chronic cannabis use is associated with abberant reward and regulatory control processes (Everitt and Robbins, 2016; Weinstein et al., 2016; Wrege et al., 2014; Yanes et al., 2018) we expected to observe corresponding maladaptations on the level of the network level organization of the brain. Specifically we hypothesized that (1) global connectivity differences between the groups specifically map to the dorsal and ventral striatum, reflecting regional-specific striatal adaptations, and (2) a shift in the network communication of the striatal subregions reflecting increased communication within fronto-striatal pathways related to reward processing and concomitantly decreased communication in pathways related to cognitive control.

\section{MATERIALS and METHODS}

\subsection{Participants}

Participants in the present study partly overlapped with the data presented in (Zimmermann et al., 2017b). To enhance the statistical power sample size was increased to $n=28$ male cannabis-dependent subjects and $n=28$ non-using controls. To control for potential confounding influences of menstrual cycle on striatal resting state activity as well as complex interactions between menstrual cycle and addiction-related alterations in fronto-striatal functioning (Franklin et al., 2015; Wetherill et al., 2016; Wiers et al., 2016), only male subjects were included (for a similar approach see Zimmermann et al., 2017a). 
All cannabis users were diagnosed with a current cannabis dependence according to DSM IV during the 18 months before the examination. To facilitate the determination of long-term alterations associated with cannabis dependence that persist beyond subacute cannabinoid metabolites (Vandevenne et al., 2000), craving (Copersino et al., 2006) and rapid neural recovery (Hirvonen et al., 2012) participants were required to abstain from cannabis for 28 days prior to the assessment.

At the time point of recruitment, most cannabis-dependent subjects were active cannabis users or in early phases of abstinence. Inclusion criteria for all participants were: (1) age between 18 and 35 years, (2) right-handedness, and (3) negative urine toxicology for cannabis and other illicit drugs (immunoassay, substance/cut-off per ml: THC/50ng, amphetamines/500ng, cocaine/300ng, methamphetamine/500ng, MDMA/300ng, opiates/300ng, methadone/300ng). Exclusion criteria for all participants were: (1) profound DSM-IV axis I or axis II disorders, such as psychotic or bipolar symptoms, (2) Beck Depression Inventory (BDI-II) score > 20 indicating a moderate depression, (3) medical disorder, (4) current/regular medication, and (5) use of other illicit substances $>75$ lifetime occasions. One user reported having used cannabis on one occasion 14 days before the experiment, but was included due to a negative urine toxicology. Controls were included if their cumulative lifetime use of cannabis was $<15 \mathrm{~g}$.

Anxiety, mood and concentration were assessed on the day of the examination using validated scales including the social interaction anxiety scale (SIAS) (Mattick and Clarke, 1998), the state-trait-anxiety inventory (STAI) (Spielberger, 1989), positive and negative affect schedule (PANAS) (Crawford and Henry, 2004) and the d2 test of attention (Brickenkamp and Zillmer, 1998). These measures allowed control for potential confounding effects of common withdrawal symptoms. Differences in potential confounders were examined by independent sample t-test; in case of a non-normal distribution of the underlying data non-parametric analyses were used.

Cannabis-dependent subjects were recruited in cooperation with the LVR Clinics Bonn, Germany, Addiction Department that offers a specialized treatment program for cannabis dependence. Written informed consent was obtained and study protocols were approved by the local ethics committee (Medical Faculty, University of Bonn), adhered to the latest 
revision of the Declaration of Helsinki and were pre-registered at clinicaltrials.gov (NCT02711371).

Three cannabis users were excluded due to a history of exceeding co-use of other illicit drugs and one was excluded due to excessive head movement during $\mathrm{fMRI}$, leading to a final sample of $n=24$ cannabis users and $n=28$ healthy controls.

\subsection{MRI Data acquisition}

Functional and structural MRI was acquired using a Siemens TRIO 3-Tesla system with a 32-channel head coil. Resting-state fMRI data was acquired before any tasks using a T2*-weighted echo-planar imaging (EPI) pulse sequence (repetition time $=2580 \mathrm{~ms}$, echo time $=30 \mathrm{~ms}$, number of slices $=47$, slice thickness $=3.5 \mathrm{~mm}$, no gap, field of view $=$ $224 \times 224 \mathrm{~mm} 2$, resolution $=64 \times 64$, flip angle $=80^{\circ}$, number of volumes $\left.=180\right)$. To improve spatial normalization and exclude participants with apparent brain pathologies a high-resolution whole-brain volume T1-weighted images was acquired in addition using a 3D spoiled gradient echo pulse sequence $($ repetition time $=1660 \mathrm{~ms}$, echo time $=2.54 \mathrm{~ms}$, flip angle $=9^{\circ}$, field of view $=256 \times 256 \mathrm{~mm} 2$, acquisition matrix $=256 \times 256$, thickness $=0.8 \mathrm{~mm}$, number of slices $\mathbf{2} 208$ ).

Participants were instructed to lie still and relax with their eyes closed while thinking of nothing in particular, yet not to fall asleep during the resting state acquisition.

\subsection{Preprocessing of the functional resting state data}

Resting state fMRI data was preprocessed using standard SPM, AFNI and FSL routines in combination with advanced independent component analysis (ICA-AROMA, Pruim et al., 2015b). The first five volumes were discarded to allow MRI T1 equilibration. The remaining volumes were slice-time corrected, spatially realigned to the first volume, and unwarped to correct for nonlinear distortions possibly related to head motion or magnetic field inhomogeneity using SPM12 (http://www.fil.ion.ucl.ac.uk/spm/software/spm12/). The functional time-series were further processed using the FMRIB Software Library (FSL, http://www.fmrib.ox.ac.uk/fsl), including non-brain removal using BET (Smith, 2002), spatial smoothing with a Gaussian kernel of $6 \mathrm{~mm}$ full width at half maximum, and grand-mean 
scaling. Subsequently, the data was submitted to an independent component analysis for automatic removal of motion artifacts (ICA-AROMA, Pruim et al., 2015b) - a procedure that has been shown to minimize the impact of motion on functional connectivity metrics and decrease the loss in temporal degrees of freedom compared to spike regression and scrubbing (Pruim et al., 2015a). Next, mean signals from white matter (WM) and cerebrospinal fluid (CSF), linear and quadratic drift across time, together with a series of sine and cosine functions removing all frequencies outside the range $(0.01-0.08 \mathrm{~Hz})$, were regressed out in a single regression step using AFNI's 3dTproject. Similar to the preprocessing protocol used by Pruim et al. (2015b), WM and CSF time-series were derived by determining the mean time-series over voxels within predefined subject-specific WM and CSF masks. To obtain these masks, we first thresholded the MNI152 average CSF and WM priors maps (95\% of the robust range) and subsequently registered to native EPI space. Likewise, we applied FSL FAST (Zhang et al., 2001) to the individual T1 images to derive a CSF and WM probability map and thresholded at $95 \%$ followed by registration to native EPI space. Multiplication of both masks resulted in the respective conservative CSF and WM masks. Finally, functional MRI data were registered to T1 and standard MNI152 space using Boundary-Based Registration as implemented in FLIRT (Jenkinson et al., 2002; Jenkinson and Smith, 2001) and FNIRT nonlinear registration (Andersson et al., 2007) with 6 and $12 \mathrm{df}$, respectively and interpolated to $2 \times 2 \times 2 \mathrm{~mm}^{3}$.

\subsection{Quality control for motion artifacts}

One participant (cannabis user) was excluded due to excessive head movement ( $>2.5 \mathrm{~mm}$ or $2.5^{\circ}$ absolute motion over the whole scan). Mean-frame-wise displacement (FD, Power et al., 2012) and spike control was employed for data quality assurance and motion control. FD was calculated according to with the raw functional data for the remaining participants.

Importantly, the two groups (controls and cannabis users) did not differ in mean FD (controls: Mean $\pm S D=0.087 \pm 0.032$, range from 0.044 to 0.145 ; cannabis users: Mean $\pm S D=0.083 \pm$ 0.024 , range from 0.042 to $0.142, p=0.568$, Cohen's $d=0.160$ ). We also defined a spike in head movements as FD $>0.3 \mathrm{~mm}$. Less than $7 \%$ scans were regarded as spikes in all of the participants and the two groups did not differ in the number of spikes (control: Mean $\pm S D=$ 
$2.427 \pm 3.501$, range from 0 to 11 ; user: Mean $\pm S D=1.750 \pm 2.723$, range from 0 to $11 . p=$ 0.445 , Cohen's d $=0.214$ ).

\subsection{Determining altered striatal connectivity in cannabis dependence using the Intrinsic}

\section{Connectivity Contrast (ICC)}

To promote an unbiased determination of striatal subregions exhibiting altered functional connectivity related to cannabis dependence, a data-driven network level approach was implemented (Walpola et al., 2017). To this end, whole-brain voxel-to-voxel connectivity was computed for each striatal voxel using the intrinsic connectivity contrast (ICC), an index similar to "degree" in graph theory but without the need for a correlation threshold, which reflected the average $r^{2}$ of a given voxel with all other brain voxels (Martuzzi et al., 2011; Rubinov and Sporns, 2010). The corresponding ICC analysis was implemented by weighting the connections of a given voxel with every other voxel in the brain (restricted to the SPM gray matter mask $>0.3$ ) by their $r^{2}$ value (Martuzzi et al., 2011), thus both negative and positive correlations will be captured in positive ICC values.

Next, resulting ICC maps were used for discriminating between cannabis-dependent subjects and healthy controls using a multivoxel pattern analysis (MVPA) with a linear kernel support vector classifier as implemented in LIBSVM (www.csie.ntu.edu.tw/ cjlin/libsvm). A searchlight procedure with a three-voxel radius was used to provide measures of classification accuracy in the neighborhood of each voxel in the striatum mask which was obtained from the Harvard-Oxford Subcortical Structural Atlases (subcortical HO atlas, Harvard Center for Morphometric Analysis) by combining the accumbens, caudate, putamen and pallidum masks (thresholded at $0 \%$ population probability) that were further masked by the gray matter mask to remove voxels with low gray matter signal. Classification was evaluated by a ten-fold cross-validation during which all participants were randomly assigned to 10 -subsamples of 5 or 6 participants using MATLAB's cvpartition function. In each iteration, the optimal hyperplane was computed based on the multivariate pattern of ICC values of 47 or 46 participants and evaluated by the excluded 5 or 6 participants. The default cost parameter $(C=1)$ was automatically corrected for unbalanced groups by adjusting weights inversely proportional to group frequencies in 
the training data. The iteration was repeated 10 times with each group being the testing set once and then the average classification accuracy of each sphere was assigned to the center voxel in the sphere. The training set was scaled to $[-1,1]$, and the testing set was scaled using the same scaling factors before applying SVM (Hsu et al., 2003). To avoid a potential bias of train-test splits, the cross-validation procedure was repeated 5 times by producing different splits in each repetition and the resultant maps were averaged to produce a convergent estimation. To test whether the resultant measures exceeded chance level, we used permutation tests to simulate the probability distribution of the classification. Briefly, we randomly shuffled the group labels and re-computed these measures (repeated 5,000 times) to build empirical distributions. The resultant maps were then converted to $p$ values and family-wise error (FWE) corrected for multiple comparisons.

\subsection{Follow-up seed-to-voxel functional connectivity}

Given that ICC is predominantly an exploratory strategy that allows a data-driven precise mapping of striatal regions characterized by their functional connectivity profile differences between the groups functional connectivity analysis was used to determine the associated networks that contribute to these differences (for a similar approach see Martuzzi et al., 2011; Walpola et al., 2017). Importantly, the rationale for the present analyses was based on addiction animal models that indicate a ventral-to-dorsal shift in striatal processing underlying the development of addiction (Everitt and Robbins, 2016). In line with our expectations, the MVPA on the ICC revealed group differences in the global connectivity profiles of ventral and dorsal striatal regions (details see results section). Consequently, the major aim of the follow-up functional connectivity analysis was to determine regions that shift communication with ventral vs dorsal striatal nodes in drug dependence. To this end, two 6-mm-radius spheres (masked by the striatum mask and the gray matter mask) centered at the peak voxels derived from the MVPA results (dorsal, ventral striatum) were used as seed regions, and Fisher Z-transformed image maps were then created by calculating the correlation coefficient of each voxel in the brain with the mean time-series of the seed regions. To determine the shift in ventral vs dorsal striatal networks associated with dependence, the subject-level seed-specific Z-maps were submitted to a 2-way mixed 
ANOVA with seed (VS and DS) entered as a within-subject factor and group (controls and cannabis users) entered as a between-subject factor employed in the FSL's Randomise Tool. Corrections for multiple comparisons were conducted using permutation-based inferences (10,000 permutations) with Threshold-Free Clustering Enhancement (TFCE) which provides a strict control while improving replicability (Chen et al., 2017; Smith and Nichols, 2009). For regions showing significant interaction effects (F-test), the mean Fisher z-transformed correlation coefficients were extracted from the underlying anatomical regions for further post hoc analysis within SPSS.

\subsection{Validation using literature-based ventral and dorsal striatal seeds}

In addition, to validate the seed-to-voxel functional connectivity results, functional connectivity analyses were then rerun using predefined VS and DS masks. An anatomical VS mask was defined as nucleus accumbens (subcortical $\mathrm{HO}$ atlas) with the probability threshold set to $30 \%$ (228 voxels in total). Following Tinnermann et al. (2017), coordinates from different studies (Daw et al., 2006; Fitzgerald et al., 2011; Luijten et al., 2017; Schönberg et al., 2007; Sweitzer et al., 2016) were averaged to create the mean coordinates of reported DS peak voxels. For those lateralized coordinates we multiplied $x$ coordinates by -1 to cover both hemispheres. The DS mask was then created by drawing two 6-mm-radius spheres centered at $\pm 13,11,14$ (for bilateral DS) and masked by the striatum mask (223 voxels in total). To control for non-gray matter signal, including WM and CSF, both VS and DS masks were masked by the gray matter mask.

\subsection{Functional characterization of the determined networks}

To support the functional characterization of the networks that exhibit a dependence-associated shift in VS versus DS communication, the NeuroSynth decoder function (http://neurosynth.org/decode/) was used to employ a large-scale automated meta-analysis map. The top 25 terms (excluding terms for brain regions) ranked by the correlation strengths between the regions exhibiting dependence-associated alterations and the meta-analytic map were visualized using word cloud with the size of the font scaled by correlation strength. 


\section{RESULTS}

\subsection{Group Characteristics}

The groups were comparable with respect to important potential confounders including socio-demographics, attention and the use of licit drugs (Table 1). The lack of significant group differences in attention, mood and state anxiety (Table 1) argues against strong confounding effects of cannabis withdrawal. As expected cannabis users reported greater lifetime experiences with other illicit drugs (Table 1) than controls. Cannabis use parameters are reported in Table 2. In post-MRI interviews none of the subjects reported having fallen asleep during the scan.

\begin{tabular}{|c|c|c|c|}
\hline Measure & $\begin{array}{c}\text { Marijuana Users } \\
\text { M (SD) }\end{array}$ & $\begin{array}{l}\text { Controls } \\
\text { M (SD) }\end{array}$ & p \\
\hline Age (years) & $24.00(3.46)$ & $23.39(2.86)$ & 0.49 \\
\hline Education (years) & $14.50(11.00-22.00)^{*}$ & $14.25(12.00-19.00)^{*}$ & $0.75^{\circ}$ \\
\hline d2 concentration performance & $196.46(39.61)$ & $205.43(50.57)$ & 0.49 \\
\hline STAI-state & $34.17(7.70)$ & $31.07(7.71)$ & 0.16 \\
\hline STAI-trait & $35.87(8.40)$ & $32.64(7.22)$ & 0.14 \\
\hline SIAS & $16.50(8.00-46.00)^{*}$ & $16.00(5.00-33.00)^{*}$ & $0.24^{\circ}$ \\
\hline Number of smokers & $N=22$ & $N=23$ & \\
\hline Years of nicotine use & $8.23(4.49)$ & $6.24(3.72)$ & 0.11 \\
\hline Cigarettes per day & $8.00(2-20.00)^{*}$ & $8.00(1-30.00)^{*}$ & $0.96^{\circ}$ \\
\hline Pack-year & $4.16(3.59)$ & $3.42(3.84)$ & 0.50 \\
\hline Alcohol occasions per week & $1.00(0-4.00)^{*}$ & $1.00(0-4.00)^{*}$ & $0.74^{a}$ \\
\hline Alcohol units per week & $5.00(0-24.00)^{*}$ & $4.90(0-18.00)^{*}$ & $0.92^{\circ}$ \\
\hline PANAS-positive & $33.00(16.00-42.00)^{*}$ & $32.50(22.00-48.00)$ & $0.67^{\circ}$ \\
\hline PANAS-negative & $11.00(10.00-25.00)^{*}$ & $10.00(10.00-14.00)^{*}$ & $0.10^{\mathrm{a}}$ \\
\hline Past ecstasy use & $N=15$ & $N=2$ & \\
\hline Lifetime occasions ecstasy & $10.00(1-75)^{*}$ & $(1,8)$ & - \\
\hline Past cocaine use & $N=11$ & $\mathrm{~N}=0$ & \\
\hline Lifetime occasions cocaine & $6.00(1-70)^{*}$ & - & \\
\hline
\end{tabular}


Past amphetamine use

Lifetime occasions amphetamine

Past hallucinogen use

Lifetime amount hallucinogen

Past opiate use

Lifetime occasions opiate

Past marijuana use

\% Lifetime marijuana dependence

$$
\begin{gathered}
N=15 \\
20.00(1-75)^{*} \\
N=9 \\
6.00(1-50)^{*} \\
N=3 \\
(1,1,4) \\
N=24 \\
100 \%
\end{gathered}
$$

$\mathrm{N}=1$

30.00

$\mathrm{N}=0$

$\mathrm{N}=1$

30.00**

$\mathrm{N}=22$

$0 \%$

Table 1. Group characteristics and drug use parameters. ${ }^{a}$ Mann-Whitney-U test, *Median(Range), ** Prescription medicinal use

\begin{tabular}{l|l}
\hline Marijuana Use Parameter & Mean \pm SD (range) (N = 24) \\
\hline Age of first marijuana use & $15.17 \pm 1.23(13-17)$ \\
Days since last marijuana use & $30.00 *(14-500)$ \\
Frequency of marijuana use (days per & $28.08 \pm 4.51(14-30)$ \\
month) & $78.38 \pm 36.40(19-144)$ \\
Duration of regular marijuana use (months) & $1616.5 *(62-5786)$ \\
\hline
\end{tabular}

Table 2. Marijuana use parameters. * Median

\subsection{Intrinsic connectivity contrast}

The searchlight MVPA identified brain regions within the striatum that exhibit altered global connectivity patterns in cannabis users compared to controls. Specifically the groups could be reliably discriminated in two regions that mapped onto the VS (peak voxel coordinates, $10,12,-10$ mapping onto the right nucleus accumbens, accuracy $=84.62 \%$, voxel-wise FWE-corrected $p=0.04$ ) and the DS (peak voxel coordinates, $-12,14,16$ mapping onto the left caudate nucleus, accuracy $=85.38 \%$, voxel-wise FWE-corrected $p=0.026$ ) (Figure 1). 


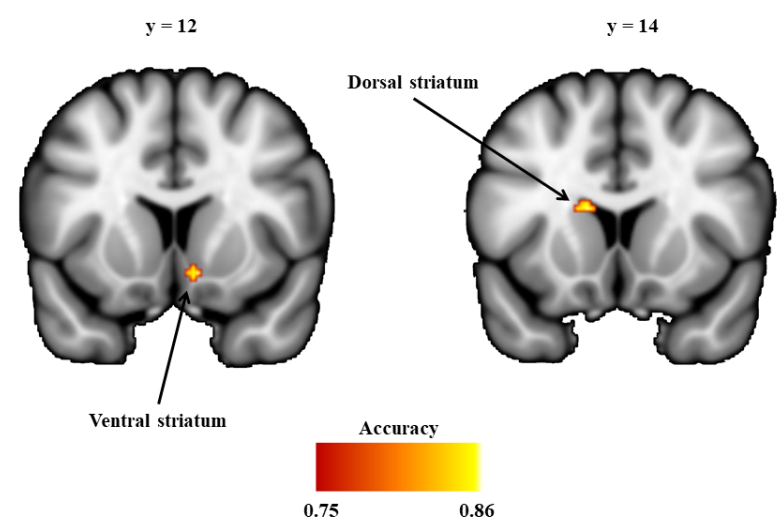

Figure 1 Striatum regions discriminating cannabis dependent subjects and non-using controls. Images are thresholded at accuracy $>=0.75$ (approximate $p<0.001$, uncorrected) and cluster size $>5$ for display purpose.

\subsection{Follow-up analyses - seed-to-voxel functional connectivity}

The main effects of seed reflecting the differential connectivity patterns of the VS vs DS seeds further confirmed the mapping of the MVPA to the VS and DS: (1) the VS seed showed stronger connectivity with ventral regions of the frontal cortex including rostal ACC and orbitofrontal regions, whereas the DS seed exhibited stronger connectivity with the dorsal frontal regions including dorsolateral prefrontal cortex, a pattern resembling previous human and animal research (Di Martino et al., 2008; Haber, 2016). (2) There was a strong overlap between the networks identified for the VS and DS regions determined in the present study and the literature-based seeds from the validation analysis (Figure 2). 


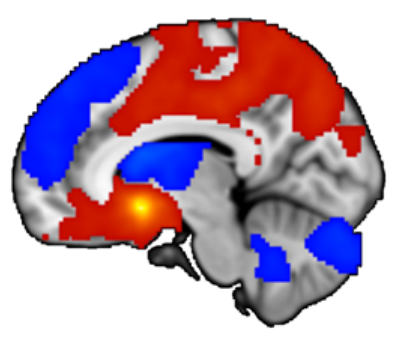

c

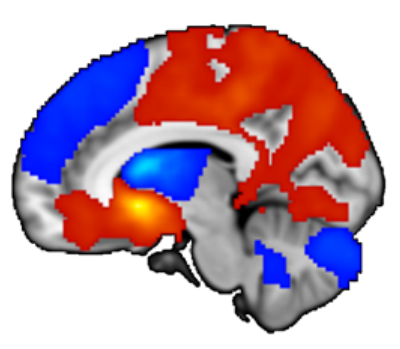

b

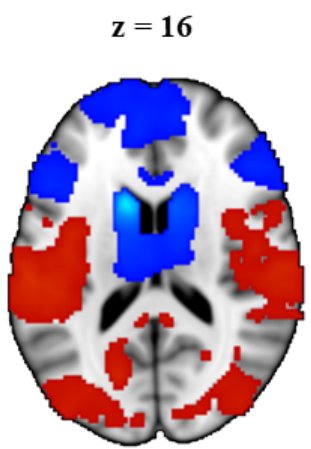

d

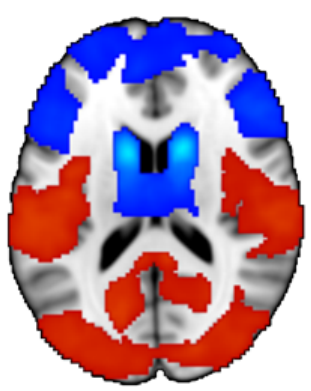

Figure 2 Brain regions showing significant main effect of seed on functional connectivity. (a) and (b) are results from data-driven seeds and (c) and (d) are results from validation seeds. Red color indicated stronger functional connectivity with VS than DS and blue color indicated stronger functional connectivity with DS than VS. All images were thresholded at $p<0.05$, TFCE corrected.

Examining differences in seed-based functional connectivity between the groups revealed no regions showing a significant main effect of group, arguing against unspecific striatal network changes in cannabis dependence. However, significant group $\mathrm{x}$ seed interaction effects were found mirroring a shift in network level communication of the striatal subregions in cannabis dependence. The interaction effects were observed in connectivity with the dorsomedial prefrontal cortex ( $\mathrm{dmPFC}$ ) and rostral anterior cingulate cortex (rACC) across both analyses (Table 3 and Figure 3).

\begin{tabular}{ccccccccc}
\hline $\mathbf{x}$ & $\mathbf{y}$ & $\mathbf{z}$ & $\begin{array}{c}\text { (sub)Peak } \\
\text { voxel F-value }\end{array}$ & $\begin{array}{c}\boldsymbol{P} \text { value (TFCE } \\
\text { corrected) }\end{array}$ & $\mathbf{k}$ & BA & Laterality & Label \\
\hline \hline \multicolumn{7}{c}{ Data-driven Seeds } \\
\hline 2 & 52 & 42 & 24.22 & 0.020 & 376 & $9 / 32 / 24$ & L/R & dmPFC \\
10 & 36 & 2 & 23.40 & 0.022 & & & & rACC \\
10 & 30 & 48 & 21.42 & 0.026 & 163 & 8 & $R$ & dmPFC \\
6 & 44 & 54 & 16.48 & 0.039 & & & & dmPFC \\
-2 & 24 & 64 & 15.14 & 0.049 & 2 & NA & L & dmPFC \\
\hline \hline
\end{tabular}




\begin{tabular}{cccccccc}
\hline \multicolumn{7}{c}{ Validation Seeds } \\
\hline \hline-6 & 44 & 20 & 21.86 & 0.004 & & $8 / 9 / 10 / 2$ & rACC \\
12 & 38 & 2 & 27.39 & 0.005 & 2147 & $4 / 32$ & L/R \\
2 & 52 & 42 & 26.29 & 0.007 & & & dmPFC \\
\hline
\end{tabular}

Table 3. Brain regions showing significant group (control vs user) by seed (ventral striatum vs dorsal striatum) interaction. $x, y, z$ : MNI-coordinates; $k$ : cluster size; BA: brodmann area; dmPFC: dorsomedial prefrontal cortex; rACC: rostral anterior cingulate cortex.

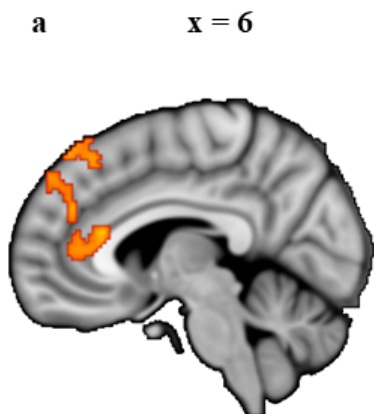

c

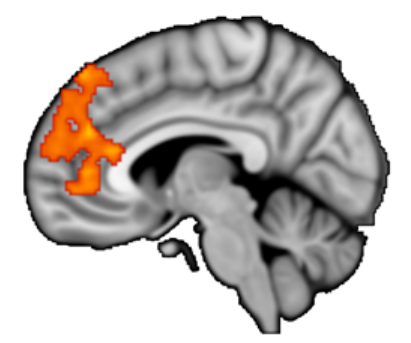

b $\quad \mathbf{x}=\mathbf{8}$

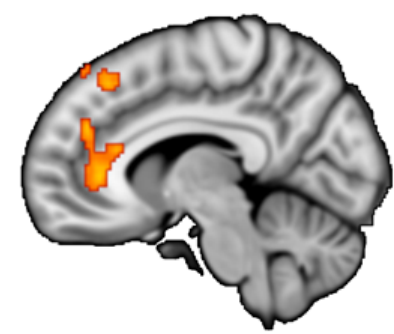

d

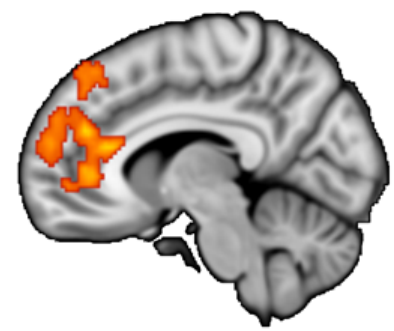

Figure 3. Brain regions showing significant group (control vs user) by seed (ventral vs dorsal striatum) functional connectivity interaction. (a) and (b) are results from data-driven seeds and (c) and (d) are results from validation seeds. All images are thresholded at $p<0.05$, TFCE corrected

\subsection{Post hoc analyses}

To further disentangle the direction of the changes using post hoc comparisons, mean Fisher z-transformed correlation coefficients were extracted from anatomical masks referring to the dmPFC and rACC. Masks of the respective regions were defined using structurally defined regions. To obtain the rACC mask, we first thresholded the anterior cingulate gyrus ( $\mathrm{HO}$ atlas) at $30 \%$ to remove voxels with low probability and the posterior boundary was then delineated as one slice anterior to the coronal plane where the connection of the corpus callosum in each hemisphere was no longer connected (Asami et al., 2008; McCormick et al., 2006). Similar, to obtain the DMPFC mask, we thresholded the superior frontal gyrus (HO atlas) at 30\% probability, excluding voxels further than $10 \mathrm{~mm}$ 
from the midline of the brain (de la Vega et al., 2016) or posterior to the anterior boundary of supplementary motor area. As displayed in Figure $4 \mathbf{a}$ and $4 \mathbf{c}$, post hoc pairwise comparisons revealed attenuated negative correlation (anti-correlation) between dmPFC and VS $\left(t_{(50)}=3.23, p=0.002\right.$, Cohen's $\left.d=0.901\right)$, as well as decreased positive connectivity between dmPFC and DS ( $t_{(50)}=-2.33, p=0.024$, Cohen's $\left.d=0.649\right)$ in cannabis users relative to controls. Post hoc comparisons on the rACC demonstrated increased positive functional connectivity with the VS $\left(t_{(50)}=2.32, p=0.025\right.$, Cohen's $\left.d=0.644\right)$ and concomitantly decreased positive functional connectivity with the DS $\left(t_{(50)}=-2.69, p=0.009\right.$, Cohen's $d=$ $0.751)$ in cannabis users compared to controls.

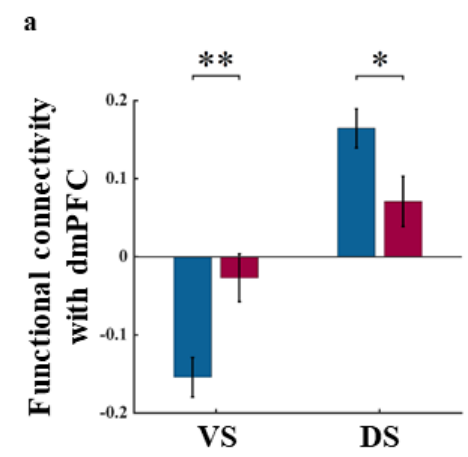

b
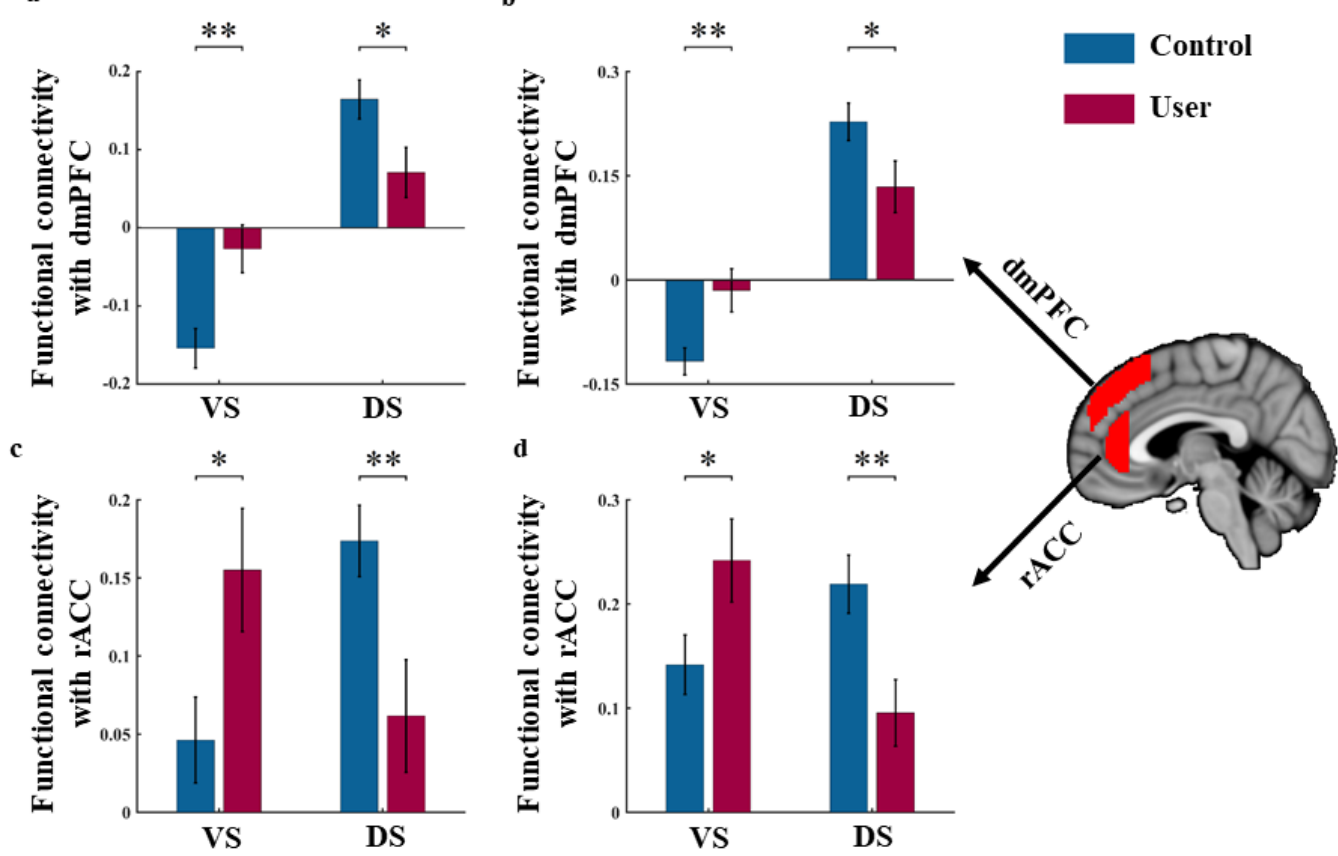

Figure 4. Patterns of functional connectivity associated with a significant group (control vs user) by seed (VS vs DS) interaction. (a) and (c) are results from data-driven seeds and (b) and (d) are results from validation seeds. Error bars reflect the SEM. ${ }^{*} p<0.05$; ${ }^{* *} p<0.01$; VS: ventral striatum; DS: dorsal striatum; dmPFC: dorsomedial prefrontal cortex; rACC: rostral anterior cingulate cortex.

\subsection{Control analyses}


Importantly none of the cannabis users fulfilled a dependence for other illicit drugs, and cannabis was their main drug of abuse. However, in line with an increased prevalence of illicit drug use in addicted populations the cannabis group had higher experience with recreational use of other drugs, particularly amphetamine (approx.. 60\%). Given that previous studies reported altered striatal functioning in occasional amphetamine users (Schrantee et al., 2016), we additionally compared the altered neural indices between users with a history of amphetamine use versus no history. This additional control analysis did not reveal significant between group differences (all ps $>0.2$ ), arguing against recreational amphetamine use as driver of the observed difference with the control group. Moreover, the control analysis with the literature-derived validation seeds revealed comparable results as the data-driven seeds, further confirming the robustness of the findings (Figure $4 b$ and $4 d$ ).

\subsection{Functional characterization of the altered networks}

NeuroSynth decoding revealed that terms showing highest correlations with the dmPFC were predominantly referring to control-related or reasoning-related processes (Figure 5a), while a predominance of reward-related or default network processes (Figure $5 \mathbf{b}$ ) was found for the rACC.

$\mathbf{a}$

\section{domain general}

\section{social}

inference

reasoning mentalizing

uncertainty consequences person beliefs task positive

\section{situation}

b

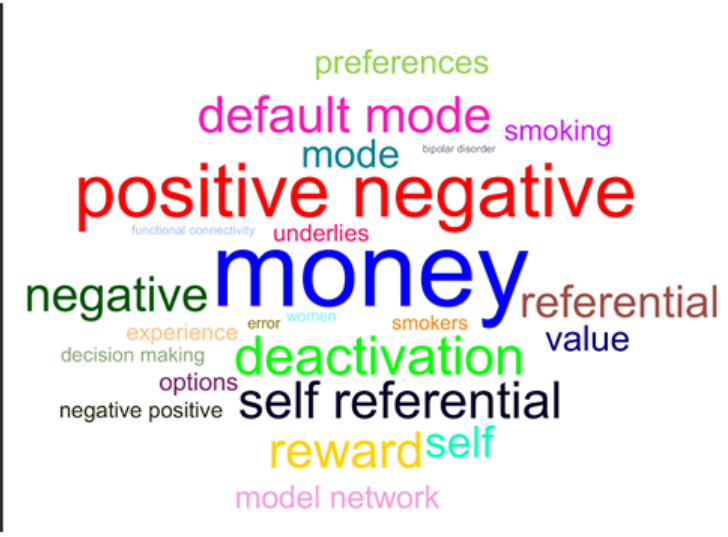

Figure 5. Word cloud showing the correlation strength between the ROIs and the meta-analytic map. (a) The top 25 terms associated with dmPFC based on Neurosynth decoding and font size represents relative correlation strength of that term to dmPFC. (b) The same information for rACC. 


\subsection{Association between cannabis use parameters and neural alterations}

Exploring associations between neural alterations and the age of onset of cannabis use, cumulative exposure and duration of abstinence (days since last use) did not reveal significant associations (all ps $>0.1$ ).

\section{DISCUSSION}

The present study combined an innovative network level approach with resting state fMRI to determine regional-specific striatal alterations and associated changes in functional communication in abstinent cannabis-dependent subjects. In line with animal and human data that emphasize the specific contributions of the VS and DS, subregion-specific differences between cannabis users and controls mapped to the nucleus accumbens and the caudate nucleus. Cannabis users displayed a relative shift between VS and DS communication with fronto-limbic regions that have been consistently involved in reward processing ( $\mathrm{rACC}$ ) and executive / regulatory functions (dmPFC). Aberrant fronto-striatal functional connectivity in the abstinent cannabis users was observed in the absence of a significant main effect in the comparison with the control group. This observation is partly in line with a previous study reporting that altered fronto-striatal connectivity in chronic cannabis users normalizes during 28 days of abstinence (Blanco-Hinojo et al., 2016). However, comparing the shift between the ventral and dorsal striatal functional networks in the present study revealed evidence for a persisting imbalance in the subregion specific fronto-striatal circuits. Together, these findings argue against unspecific long-term striatal changes in cannabis dependence but rather emphasize the importance of both, specific adaptations in dorsal and ventral subregions and a relative shift between the regions.

Moreover, employing a fully-data driven network approach that combines the intrinsic connectivity contrast with a pattern classification demonstrated that the general connectivity patterns of the VS and DS can reliably discriminate between cannabis-dependent individuals and controls. As such, the present data are in line with previous studies reporting altered ventral and dorsal striatal activity in chronic cannabis users during drug cue reactivity, reward processing (Weinstein et al., 2016; Wrege et al., 
2014; Yanes et al., 2018) and social decision making (Gilman, 2017). Further, our data emphasize the important contribution of striatal maladaptations in addiction which might reflect a common pathological pathway across addictive disorders. In accordance with a plethora of previous animal and human research suggesting a functional differentiation of the striatum along a dorsal to ventral gradient (Delgado, 2007) and a specific contribution of the striatal subregions to the transition to addiction (Everitt and Robbins, 2016), differences in the intrinsic function of both subregions were demonstrated. Specifically, the nucleus accumbens, a core reward processing node (Delgado, 2007), and the caudate nucleus, strongly engaged in cognitive processes and executive control (Haber, 2016), differed between both groups.

The mapping of the identified regions and their proposed functional contributions was further confirmed by the stronger connectivity of the VS with reward-related limbic and (orbito-)frontal regions, whereas the DS showed a stronger functional interaction with frontal regions such as the dorsolateral prefrontal cortex (dIPFC) that are critically involved in cognitive and regulatory processes. Examining differences in the connectivity profiles of the identified VS and DS regions revealed a relative shift in the connectivity with the dmPFC, a region that has been implicated in regulatory downstream control over the striatum (Kragel et al., 2018; Robbins et al., 2012) and the rACC, a region engaged in reward processing via up-stream signaling of the VS (Haber and Knutson, 2010). As such, the present findings are in accordance with recent quantitative and qualitative reviews suggesting alterations in networks engaged in cognitive control and reward processing in chronic cannabis users (Weinstein et al., 2016; Wrege et al., 2014; Yanes et al., 2018).

More specifically, both striatal regions demonstrated an uncoupling with dmPFC regulatory regions which may reflect deficient inhibitory control which is consistently observed in cannabis users (Wrege et al., 2014). The altered caudate-dmPFC pathway suggests specific deficits in stop-signal inhibition (Robbins et al., 2012), an inhibitory domain that has been shown to be deficient across addictive disorders (Acikalin et al., 2017; Morein-Zamir et al., 2013). By contrast, in cannabis-dependent subjects the rACC exhibited increased connectivity with the VS and concomitantly decreased connectivity with the DS. Increased connectivity in the nucleus accumbens - ACC pathway in response to drug cues 
has previously been identified as a specific marker for dependent versus non-dependent cannabis users (Filbey and Dunlop, 2014). Activation in the rACC and the adjacent ventromedial PFC have been found to reliably reflect subjective value and reward representations (Acikalin et al., 2017). The pathway with the VS has been associated with the degree of delayed discounting-associated inhibitory control (Li et al., 2013; see also circuit model by Robbins et al., 2012) and deficits in this domain have also been observed in cocaine-dependent individuals (Contreras - Rodríguez et al., 2015). Consistent with the present results on decreased DS-rACC connectivity, a previous study reported attenuated connectivity in this pathway in nicotine-dependent individuals (Sweitzer et al., 2016). This has been interpreted as disengagement of habitual behavior in favor of conscious perception of craving that promotes drug seeking behavior. To this end, altered striatal rACC connectivity may reflect increased sensitivity to rewards as proposed by the incentive salience theory of addiction (Robinson and Berridge, 2000) which has recently been demonstrated in cannabis dependence (Filbey et al., 2016) and an increased sensitivity for internally generated negative feelings related to craving.

The present findings need to be considered in the context of limitations. To facilitate the determination of elementary fronto-striatal alterations in cannabis dependence the study focused on male participants. The rationale was based on an increasing number of studies reporting modulatory effects of menstrual cycle phase on fronto-striatal processing and associated functions (e.g. Diekhof and Ratnayake, 2016; Dreher et al., 2007) as well as recent studies suggesting that addiction-related alterations in these circuits vary as a function of menstrual cycle (Franklin et al., 2015; Wetherill et al., 2016; for cannabis see Wiers et al., 2016). The focus on a male sample allowed to control for these potential confounding variables, however, comes at a cost of a limited generalization of the present findings to cannabis dependent women. In the context of emerging evidence for a higher sensitivity of women to the adverse effects of chronic cannabis use on the brain, including blunted dopaminergic reactivity in the striatum and impaired frontal activity (Paola Castelli et al., 2014; Wiers et al., 2016), future studies need to consider to explicitly examine sex-differences, (2) due to the high prevalence of tobacco co-use in cannabis addiction both samples included a high proportion of tobacco smokers, although the groups were matched 
with respect to tobacco use parameters complex interactions between cannabis and tobacco use may have contributed to the present findings, and (3) the cannabis sample size was relatively small which limited ability to assess within-group clinical correlations.

Together the present findings suggest a shift in the balance between dorsal and ventral striatal control of behavior in cannabis dependence. Similar changes have been previously validated in comprehensive animal models of addiction and may promote the loss of control central to the transition to addictive behavior. 
bioRxiv preprint doi: https://doi.org/10.1101/282939; this version posted March 15, 2018. The copyright holder for this preprint (which was not certified by peer review) is the author/funder, who has granted bioRxiv a license to display the preprint in perpetuity. It is made available under aCC-BY-NC-ND 4.0 International license.

\section{FUNDING AND DISCLOSURE}

This work was supported by the National Natural Science Foundation of China (NSFC,

91632117; 31530032), the German Research Foundation (DFG, grant: BE5465/2-1,

HU1302/4-1), All other authors report no biomedical financial interests or potential conflicts of interest. 


\section{REFERENCES}

Acikalin, M.Y., Gorgolewski, K.J., Poldrack, R.A., 2017. A coordinate-based meta-analysis of overlaps in regional specialization and functional connectivity across subjective value and default mode networks. Frontiers in neuroscience 11, 1. Andersson, J.L., Jenkinson, M., Smith, S., 2007. Non-linear registration, aka Spatial normalisation FMRIB technical report TR07JA2. FMRIB Analysis Group of the University of Oxford 2, 1-21.

Asami, T., Hayano, F., Nakamura, M., Yamasue, H., Uehara, K., Otsuka, T., Roppongi, T., Nihashi, N., Inoue, T., Hirayasu, Y., 2008. Anterior cingulate cortex volume reduction in patients with panic disorder. Psychiatry and Clinical Neurosciences 62, 322-330.

Blanco - Hinojo, L., Pujol, J., Harrison, B.J., Macià, D., Batalla, A., Nogué, S., Torrens, M., Farré, M., Deus, J., Martín - Santos, R., 2017. Attenuated frontal and sensory inputs to the basal ganglia in cannabis users. Addiction biology 22, 1036-1047.

Brand, M., Young, K.S., Laier, C., Wölfling, K., Potenza, M.N., 2016. Integrating psychological and neurobiological considerations regarding the development and maintenance of specific Internet-use disorders: An Interaction of Person-Affect-Cognition-Execution (I-PACE) model. Neuroscience \& Biobehavioral Reviews 71, 252-266.

Brickenkamp, R., Zillmer, E., 1998. The d2 test of attention. Hogrefe \& Huber Pub. Chen, X., Lu, B., Yan, C.-G., 2017. Reproducibility of R-fMRI Metrics on the Impact of Different Strategies for Multiple Comparison Correction and Sample Sizes. bioRxiv, 128645.

Contreras - Rodríguez, O., Albein - Urios, N., Perales, J.C., Martínez - Gonzalez, J.M., Vilar - López, R., Fernández - Serrano, M.J., Lozano - Rojas, O., Verdejo García, A., 2015. Cocaine - specific neuroplasticity in the ventral striatum network is linked to delay discounting and drug relapse. Addiction 110, 1953-1962.

Copersino, M.L., Boyd, S.J., Tashkin, D.P., Huestis, M.A., Heishman, S.J., Dermand, J.C., Simmons, M.S., Gorelick, D.A., 2006. Cannabis Withdrawal Among Non Treatment - Seeking Adult Cannabis Users. The American Journal on Addictions 15, 8-14.

Crawford, J.R., Henry, J.D., 2004. The Positive and Negative Affect Schedule (PANAS): Construct validity, measurement properties and normative data in a large non - clinical sample. British journal of clinical psychology 43, 245-265.

Crean, R.D., Crane, N.A., Mason, B.J., 2011. An evidence based review of acute and long-term effects of cannabis use on executive cognitive functions. Journal of addiction medicine 5,1 .

Daw, N.D., O'doherty, J.P., Dayan, P., Seymour, B., Dolan, R.J., 2006. Cortical substrates for exploratory decisions in humans. Nature 441, 876.

Delgado, M.R., 2007. Reward - related responses in the human striatum. Ann N Y Acad Sci 1104, 70-88. 
Di Martino, A., Kelly, C., Grzadzinski, R., Zuo, X.-N., Mennes, M., Mairena, M.A., Lord, C., Castellanos, F.X., Milham, M.P., 2011. Aberrant striatal functional connectivity in children with autism. Biol Psychiatry 69, 847-856.

Di Martino, A., Scheres, A., Margulies, D.S., Kelly, A., Uddin, L.Q., Shehzad, Z., Biswal, B., Walters, J.R., Castellanos, F.X., Milham, M.P., 2008. Functional connectivity of human striatum: a resting state FMRI study. Cerebral cortex 18 , 2735-2747.

Diekhof, E.K., Ratnayake, M., 2016. Menstrual cycle phase modulates reward sensitivity and performance monitoring in young women: Preliminary fMRI evidence. Neuropsychologia 84, 70-80.

Dreher, J.-C., Schmidt, P.J., Kohn, P., Furman, D., Rubinow, D., Berman, K.F., 2007. Menstrual cycle phase modulates reward-related neural function in women.

Proceedings of the National Academy of Sciences 104, 2465-2470.

EMCDDA, 2008. European monitoring centre for drugs and drug addiction annual report.

Everitt, B.J., Robbins, T.W., 2016. Drug addiction: updating actions to habits to compulsions ten years on. Annual review of psychology 67, 23-50.

Filbey, F.M., Dunlop, J., 2014. Differential reward network functional connectivity in cannabis dependent and non-dependent users. Drug \& Alcohol Dependence 140, 101-111.

Filbey, F.M., Dunlop, J., Ketcherside, A., Baine, J., Rhinehardt, T., Kuhn, B., DeWitt, S., Alvi, T., 2016. fMRI study of neural sensitization to hedonic stimuli in long - term, daily cannabis users. Human Brain Mapping 37, 3431-3443.

Fineberg, N.A., Potenza, M.N., Chamberlain, S.R., Berlin, H.A., Menzies, L., Bechara, A., Sahakian, B.J., Robbins, T.W., Bullmore, E.T., Hollander, E., 2010.

Probing compulsive and impulsive behaviors, from animal models to endophenotypes: a narrative review. Neuropsychopharmacology 35, 591.

Fitzgerald, K.D., Welsh, R.C., Stern, E.R., Angstadt, M., Hanna, G.L., Abelson, J.L., Taylor, S.F., 2011. Developmental alterations of frontal-striatal-thalamic connectivity in obsessive-compulsive disorder. Journal of the American Academy of Child \& Adolescent Psychiatry 50, 938-948. e933.

Franklin, T.R., Jagannathan, K., Wetherill, R.R., Johnson, B., Kelly, S., Langguth, J., Mumma, J., Childress, A.R., 2015. Influence of menstrual cycle phase on neural and craving responses to appetitive smoking cues in naturally cycling females. Nicotine \& Tobacco Research 17, 390-397.

Ganzer, F., Bröning, S., Kraft, S., Sack, P.-M., Thomasius, R., 2016. Weighing the evidence: a systematic review on long-term neurocognitive effects of cannabis use in abstinent adolescents and adults. Neuropsychology review 26, 186-222.

Gilman, J.M., 2017. Neural Correlates of Social Influence Among Cannabis Users. Current addiction reports 4, 53-61.

Goldstein, R.Z., Volkow, N.D., 2011. Dysfunction of the prefrontal cortex in addiction: neuroimaging findings and clinical implications. Nature reviews neuroscience 12, 652 .

Haber, S.N., 2016. Corticostriatal circuitry. Neuroscience in the 21st Century, 1-21. 
Haber, S.N., Knutson, B., 2010. The reward circuit: linking primate anatomy and human imaging. Neuropsychopharmacology 35, 4.

Hirvonen, J., Goodwin, R., Li, C.-T., Terry, G., Zoghbi, S., Morse, C., Pike, V., Volkow, N., Huestis, M., Innis, R., 2012. Reversible and regionally selective downregulation of brain cannabinoid CB 1 receptors in chronic daily cannabis smokers. Molecular psychiatry 17, 642.

Hsu, C.-W., Chang, C.-C., Lin, C.-J., 2003. A practical guide to support vector classification.

Jager, G., Block, R.I., Luijten, M., Ramsey, N.F., 2013. Tentative evidence for striatal hyperactivity in adolescent cannabis-using boys: a cross-sectional multicenter fMRI study. Journal of psychoactive drugs 45, 156-167.

Jenkinson, M., Bannister, P., Brady, M., Smith, S., 2002. Improved optimization for the robust and accurate linear registration and motion correction of brain images.

Neuroimage 17, 825-841.

Jenkinson, M., Smith, S., 2001. A global optimisation method for robust affine registration of brain images. Medical image analysis 5, 143-156.

Jentsch, J.D., Taylor, J.R., 1999. Impulsivity resulting from frontostriatal dysfunction in drug abuse: implications for the control of behavior by reward-related stimuli. Psychopharmacology 146, 373-390.

Kragel, P.A., Kano, M., Van Oudenhove, L., Ly, H.G., Dupont, P., Rubio, A., Delon-Martin, C., Bonaz, B.L., Manuck, S.B., Gianaros, P.J., 2018. Generalizable representations of pain, cognitive control, and negative emotion in medial frontal cortex. Nat Neurosci, 1.

Li, N., Ma, N., Liu, Y., He, X.-S., Sun, D.-L., Fu, X.-M., Zhang, X., Han, S., Zhang, D.-R., 2013. Resting-state functional connectivity predicts impulsivity in economic decision-making. Journal of Neuroscience 33, 4886-4895.

Lorenzetti, V., Solowij, N., Yücel, M., 2016. The role of cannabinoids in neuroanatomic alterations in cannabis users. Biol Psychiatry 79, e17-e31.

Luijten, M., Schellekens, A.F., Kühn, S., Machielse, M.W., Sescousse, G., 2017. Disruption of reward processing in addiction: an image-based meta-analysis of functional magnetic resonance imaging studies. JAMA psychiatry 74, 387-398. Martuzzi, R., Ramani, R., Qiu, M., Shen, X., Papademetris, X., Constable, R.T., 2011. A whole-brain voxel based measure of intrinsic connectivity contrast reveals local changes in tissue connectivity with anesthetic without a priori assumptions on thresholds or regions of interest. Neuroimage 58, 1044-1050.

Mattick, R.P., Clarke, J.C., 1998. Development and validation of measures of social phobia scrutiny fear and social interaction anxiety1. Behaviour research and therapy 36, 455-470.

McCormick, L.M., Ziebell, S., Nopoulos, P., Cassell, M., Andreasen, N.C., Brumm, M., 2006. Anterior cingulate cortex: an MRI-based parcellation method. Neuroimage 32, 1167-1175.

Morein-Zamir, S., Jones, P.S., Bullmore, E.T., Robbins, T.W., Ersche, K.D., 2013. Prefrontal hypoactivity associated with impaired inhibition in stimulant-dependent 
individuals but evidence for hyperactivation in their unaffected siblings.

Neuropsychopharmacology 38, 1945.

Morein-Zamir, S., Robbins, T.W., 2015. Fronto-striatal circuits in response-inhibition:

Relevance to addiction. Brain Res 1628, 117-129.

Paola Castelli, M., Fadda, P., Casu, A., Sabrina Spano, M., Casti, A., Fratta, W., Fattore, L., 2014. Male and female rats differ in brain cannabinoid CB1 receptor density and function and in behavioural traits predisposing to drug addiction: effect of ovarian hormones. Curr Pharm Des 20, 2100-2113.

Postuma, R.B., Dagher, A., 2006. Basal ganglia functional connectivity based on a meta-analysis of 126 positron emission tomography and functional magnetic resonance imaging publications. Cerebral cortex 16, 1508-1521.

Power, J.D., Barnes, K.A., Snyder, A.Z., Schlaggar, B.L., Petersen, S.E., 2012.

Spurious but systematic correlations in functional connectivity MRI networks arise from subject motion. Neuroimage 59, 2142-2154.

Pruim, R.H., Mennes, M., Buitelaar, J.K., Beckmann, C.F., 2015a. Evaluation of ICA-AROMA and alternative strategies for motion artifact removal in resting state fMRI. Neuroimage 112, 278-287.

Pruim, R.H., Mennes, M., van Rooij, D., Llera, A., Buitelaar, J.K., Beckmann, C.F., 2015b. ICA-AROMA: A robust ICA-based strategy for removing motion artifacts from fMRI data. Neuroimage 112, 267-277.

Robbins, T.W., Gillan, C.M., Smith, D.G., de Wit, S., Ersche, K.D., 2012. Neurocognitive endophenotypes of impulsivity and compulsivity: towards dimensional psychiatry. Trends Cogn Sci 16, 81-91.

Robinson, T.E., Berridge, K.C., 2000. The psychology and neurobiology of addiction: an incentive-sensitization view. Addiction 95, 91-117.

Rubinov, M., Sporns, O., 2010. Complex network measures of brain connectivity: uses and interpretations. Neuroimage 52, 1059-1069.

Schönberg, T., Daw, N.D., Joel, D., O'Doherty, J.P., 2007. Reinforcement learning signals in the human striatum distinguish learners from nonlearners during reward-based decision making. Journal of Neuroscience 27, 12860-12867.

Schrantee, A., Ferguson, B., Stoffers, D., Booij, J., Rombouts, S., Reneman, L., 2016. Effects of dexamphetamine-induced dopamine release on resting-state network connectivity in recreational amphetamine users and healthy controls. Brain Imaging and Behavior 10, 548-558.

Schreiner, A.M., Dunn, M.E., 2012. Residual effects of cannabis use on neurocognitive performance after prolonged abstinence: a meta-analysis.

Experimental and clinical psychopharmacology 20, 420.

Smith, S.M., 2002. Fast robust automated brain extraction. Human Brain Mapping 17, 143-155.

Smith, S.M., Nichols, T.E., 2009. Threshold-free cluster enhancement: addressing problems of smoothing, threshold dependence and localisation in cluster inference. Neuroimage 44, 83-98.

Spielberger, C.D., 1989. State-trait anxiety inventory: a comprehensive bibliography. Consulting Psychologists Press. 
Sweitzer, M.M., Geier, C.F., Addicott, M.A., Denlinger, R., Raiff, B.R., Dallery, J., McClernon, F.J., Donny, E.C., 2016. Smoking abstinence-induced changes in resting state functional connectivity with ventral striatum predict lapse during a quit attempt. Neuropsychopharmacology 41, 2521.

Tinnermann, A., Geuter, S., Sprenger, C., Finsterbusch, J., Büchel, C., 2017. Interactions between brain and spinal cord mediate value effects in nocebo hyperalgesia. Science 358, 105-108.

Vandevenne, M., Vandenbussche, H., Verstraete, A., 2000. Detection time of drugs of abuse in urine. Acta Clinica Belgica 55, 323-333.

Volkow, N.D., Swanson, J.M., Evins, A.E., DeLisi, L.E., Meier, M.H., Gonzalez, R., Bloomfield, M.A., Curran, H.V., Baler, R., 2016. Effects of cannabis use on human behavior, including cognition, motivation, and psychosis: a review. JAMA psychiatry 73, 292-297.

Walpola, I.C., Nest, T., Roseman, L., Erritzoe, D., Feilding, A., Nutt, D.J., Carhart-Harris, R.L., 2017. Altered insula connectivity under MDMA.

Neuropsychopharmacology 42, 2152.

Weinstein, A., Livny, A., Weizman, A., 2016. Brain imaging studies on the cognitive, pharmacological and neurobiological effects of cannabis in humans: evidence from studies of adult users. Curr Pharm Des 22, 6366-6379.

Wetherill, R.R., Jagannathan, K., Hager, N., Maron, M., Franklin, T.R., 2016. Influence of menstrual cycle phase on resting-state functional connectivity in naturally cycling, cigarette-dependent women. Biology of sex differences 7, 24. Wiers, C.E., Shokri-Kojori, E., Wong, C.T., Abi-Dargham, A., Demiral, Ş.B., Tomasi, D., Wang, G.-J., Volkow, N.D., 2016. Cannabis abusers show hypofrontality and blunted brain responses to a stimulant challenge in females but not in males.

Neuropsychopharmacology 41, 2596.

Wrege, J., Schmidt, A., Walter, A., Smieskova, R., Bendfeldt, K., Radue, E.-W., E Lang, U., Borgwardt, S., 2014. Effects of cannabis on impulsivity: a systematic review of neuroimaging findings. Curr Pharm Des 20, 2126-2137.

Yanes, J.A., Riedel, M.C., Ray, K.L., Kirkland, A.E., Bird, R.T., Boeving, E.R., Reid, M.A., Gonzalez, R., Robinson, J.L., Laird, A.R., 2018. Neuroimaging meta-analysis of cannabis use studies reveals convergent functional alterations in brain regions supporting cognitive control and reward processing. Journal of Psychopharmacology, 0269881117744995.

Zhang, Y., Brady, M., Smith, S., 2001. Segmentation of brain MR images through a hidden Markov random field model and the expectation-maximization algorithm. IEEE transactions on medical imaging 20, 45-57.

Zimmermann, K., Walz, C., Derckx, R.T., Kendrick, K.M., Weber, B., Dore, B., Ochsner, K.N., Hurlemann, R., Becker, B., 2017a. Emotion regulation deficits in regular marijuana users. Human Brain Mapping 38, 4270-4279.

Zimmermann, K., Yao, S., Heinz, M., Zhou, F., Dau, W., Banger, M., Weber, B., Hurlemann, R., Becker, B., 2017b. Altered orbitofrontal activity and dorsal striatal connectivity during emotion processing in dependent marijuana users after 28 days of abstinence. Psychopharmacology, 1-11. 
bioRxiv preprint doi: https://doi.org/10.1101/282939; this version posted March 15, 2018. The copyright holder for this preprint (which was not certified by peer review) is the author/funder, who has granted bioRxiv a license to display the preprint in perpetuity. It is made available under aCC-BY-NC-ND 4.0 International license. 